\title{
Septate junction proteins are required for egg elongation and border cell migration during oogenesis in Drosophila
}

\author{
Haifa Alhadyian, Dania Shoaib, and Robert E. Ward IV*, \\ Department of Molecular Biosciences, University of Kansas, Lawrence, KS 66045, USA \\ ${ }^{\dagger}$ Present address: Department of Biology, Case Western Reserve University, Cleveland, OH 44106, USA. \\ *Corresponding author: Department of Biology, Case Western Reserve University, 2080 Adelbert Road, Cleveland, OH 44106, USA. Email: rew130@case.edu
}

\begin{abstract}
Protein components of the invertebrate occluding junction—known as the septate junction (SJ)_are required for morphogenetic developmental events during embryogenesis in Drosophila melanogaster. In order to determine whether SJ proteins are similarly required for morphogenesis during other developmental stages, we investigated the localization and requirement of four representative SJ proteins during oogenesis: Contactin, Macroglobulin complement-related, Neurexin IV, and Coracle. A number of morphogenetic processes occur during oogenesis, including egg elongation, formation of dorsal appendages, and border cell (BC) migration. We found that all four SJ proteins are expressed in egg chambers throughout oogenesis, with the highest and the most sustained levels in the follicular epithelium (FE). In the FE, SJ proteins localize along the lateral membrane during early and mid-oogenesis, but become enriched in an apical-lateral domain (the presumptive SJ) by stage 11. SJ protein relocalization requires the expression of other SJ proteins, as well as Rab5 and Rab11 like SJ biogenesis in the embryo. Knocking down the expression of these SJ proteins in follicle cells throughout oogenesis results in egg elongation defects and abnormal dorsal appendages. Similarly, reducing the expression of SJ genes in the BC cluster results in BC migration defects. Together, these results demonstrate an essential requirement for SJ genes in morphogenesis during oogenesis, and suggest that SJ proteins may have conserved functions in epithelial morphogenesis across developmental stages.
\end{abstract}

Keywords: septate junctions; oogenesis; Drosophila; tissue morphogenesis; egg elongation

\section{Introduction}

The septate junction (SJ) provides an essential paracellular barrier to epithelial tissues in invertebrate animals (Noirot-timothée et al. 1978). As such, the SJ is functionally equivalent to the tight junction in vertebrate tissues, although the molecular components and ultrastructure of these junctions differ (reviewed in Izumi and Furuse 2014). Studies in Drosophila have identified more than 20 proteins that are required for the organization or maintenance of the SJ (Fehon et al. 1994; Baumgartner et al. 1996; Behr et al. 2003; Genova and Fehon 2003; Paul et al. 2003; FaivreSarrailh et al. 2004; Wu et al. 2004, 2007; Nelson et al. 2010; Tiklová et al. 2010; Ile et al. 2012; Bätz et al. 2014; Hall et al. 2014). Given that some of these genes have clear developmental functions (e.g., coracle's name derives from its dorsal open embryonic phenotype; Fehon et al. 1994), we previously undertook an examination of the developmental requirements for a set of core SJ genes (Hall and Ward 2016). We found that all of the genes we analyzed (9 in all) are required for morphogenetic developmental events during embryogenesis including head involution, dorsal closure, and salivary gland organogenesis. Interestingly, these embryonic developmental events occur prior to the formation of an intact SJ, suggesting that these proteins have a function independent of their role in creating the occluding junction (Hall and Ward 2016). Because strong loss of function mutations in every SJ gene are embryonic lethal (due to these morphogenetic defects and/or a failure in establishing a blood-brain barrier in glial cells; Baumgartner et al. 1996), only a few studies have examined the role of SJ proteins in morphogenesis at later stages of development. These studies have revealed roles for SJ proteins in planar polarization of the wing imaginal disc, for epithelial rotations in the eye and genital imaginal discs, and ommatidia integrity (Lamb et al. 1998; Venema et al. 2004; Banerjee et al. 2008; Moyer and Jacobs 2008).

To further explore the role of SJ proteins in morphogenesis beyond the embryonic stage, we set out to examine the expression and function of a subset of SJ genes in the Drosophila egg chamber during oogenesis. Each of the two Drosophila ovaries is comprised of approximately 16-20 ovarioles, which are organized into strings of progressively developing egg chambers (Figure 1A). Each egg chamber forms in a structure called the germarium, where the germline and somatic stem cells reside. Once the egg chamber is formed, it leaves the germarium as a 16-cell germline cyst consisting of 15 nurse cells (NCs) and an oocyte surrounded by a layer of somatic follicle cells (FCs) (Figure 1B). An egg chamber undergoes 14 developmental stages ending in a mature egg that is ready for fertilization (reviewed in Horne-Badovinac and Bilder 2005). Interfollicular cells called stalk cells (SCs) connect egg chambers to each other. During oogenesis, the follicular epithelium (FE) participates in several morphogenetic events 

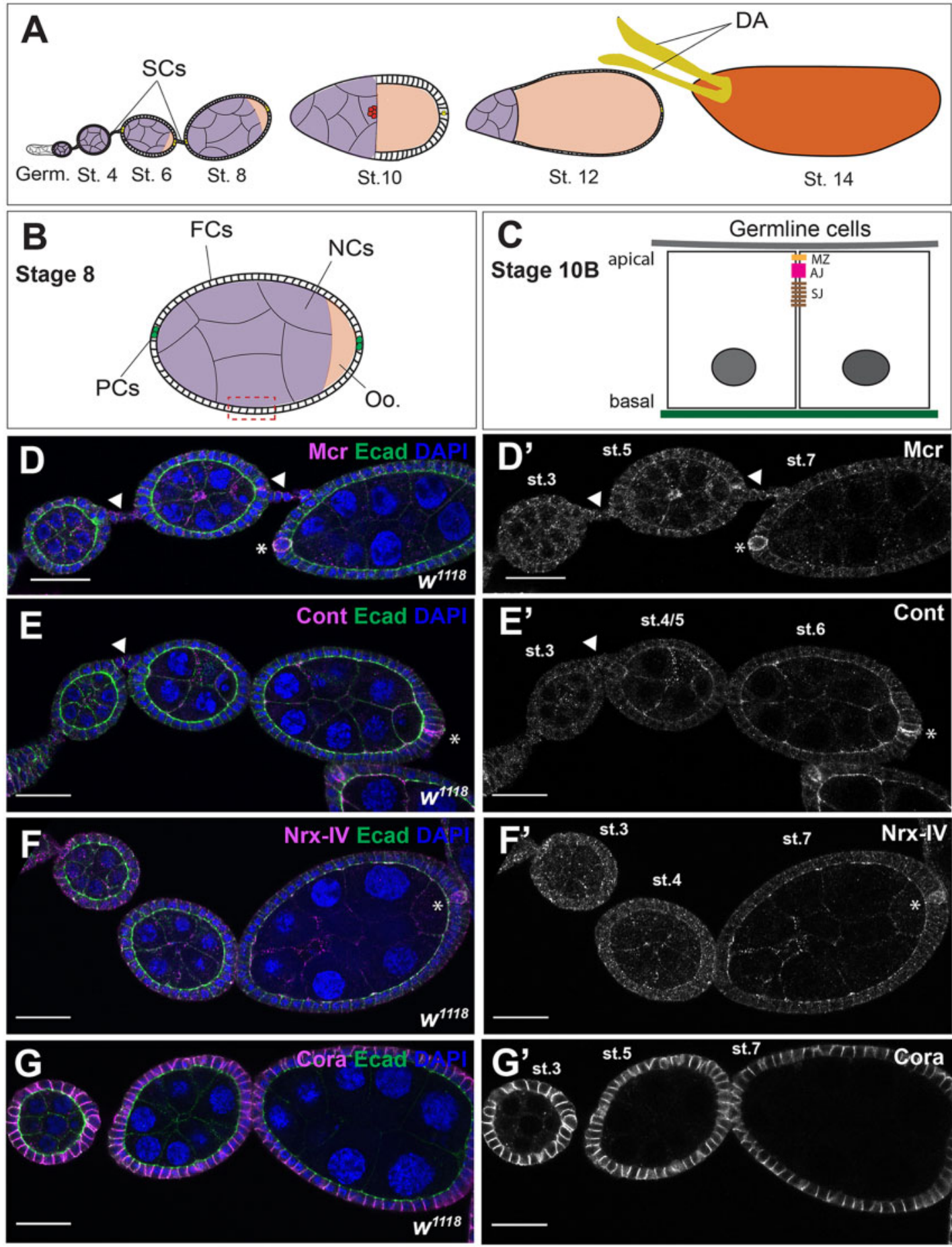

Figure $1 \mathrm{Mcr}$, Cont, Nrx-IV, and Cora expression during early stages of oogenesis. (A) Diagram of a female Drosophila ovariole. Egg chambers are formed in the most anterior region of the ovariole called the germarium (Germ). Each egg chamber undergoes 14 developmental stages while connected to each other through SCs to form a mature stage 14 egg. (B) Diagram of a stage 8 egg chamber. The egg chamber consists of 15 NCs and one oocyte (Oo.), which are surrounded by a monolayer of FCs. At the anterior and posterior ends of an egg chamber resides a pair of differentiated FCs called PCs. (C) Diagram of a lateral view of a portion of a stage 10B egg chamber. FCs face the germline and have defined apical-basal polarity with the apical surface facing the germline and a lateral junctional complex consisting of a marginal zone (MZ), an adherens junction (AJ), and a SJ. (D-G) Confocal optical sections of wild-type early stages egg chambers stained with antibodies against Mcr (D), Cont (E), Nrx-IV (F), and Cora (G) (Magenta and in individual channel in D'G'), and co-stained with antibodies against Ecad (green) and labeled with DAPI (blue). All four SJ proteins are expressed throughout the egg chamber along FC membranes, including SCs (arrowheads in D and E, and data not shown for Nrx-IV and Cora) and in the NCs. Mcr, Cont, and Nrx-IV (D-F) are found along the membrane and in puncta, whereas Cora is found predominantly at the membrane (G). In addition, Mcr, Cont, and Nrx-IV are highly expressed in the PCs (asterisks in D-F), whereas Cora is expressed in these cells with same level of expression relative to the FCs. Note that the focal plane of these images shows strong staining in PCs in only one side of the egg chamber, but Mcr, Cont, and Nrx-IV are equally expressed in both anterior and posterior PCs. Anterior is to the left in each ovariole. Scale bar $=25 \mu \mathrm{m}$. 
including border cell (BC) migration, dorsal appendage formation, and egg elongation (reviewed in Horne-Badovinac and Bilder 2005; Duhart et al. 2017).

Previous studies have revealed that a few core components of the SJ are expressed in the ovary, including Macroglobulin complement-related (Mcr), Neurexin IV (Nrx-IV), Contactin (Cont), Neuroglian (Nrg), and Coracle (Cora) (Wei et al. 2004; Schneider et al. 2006; Hall et al. 2014; Maimon et al. 2014; Ben-Zvi and Volk 2019), although the developmental expression pattern and subcellular localizations of these proteins had not been thoroughly investigated. In addition, ultrastructural analysis has revealed the presence of mature SJs in the FE by stage 10/10B of oogenesis (Figure 1C), with incipient SJ structures observed in egg chambers as early as stage 6 (Mahowald 1972; Müller 2000). How SJ maturation occurs in the FE is unknown. In embryonic epithelia, SJ biogenesis is a multistep process in which SJ proteins are initially localized along the lateral membrane, but become restricted to an apical-lateral region (the SJ) in a process that requires endocytosis and recycling of SJ proteins (Tiklová et al. 2010).

Here, we analyzed the expression and subcellular localization of the core SJ proteins Mcr, Cont, Nrx-IV, and Cora throughout oogenesis. We find that all of these SJ proteins are expressed in the FE throughout oogenesis. Interestingly, Mcr, Cont, Nrx-IV, and Cora become enriched at the most apical-lateral region of the membrane in stage 11 egg chambers, coincident with the formation of the SJ revealed by electron microscopy (Mahowald 1972; Müller 2000). Similar to the biogenesis of SJs in the embryo, this enrichment of SJ proteins to the presumptive SJ requires the function of other SJ genes, as well as Rab5 and Rab11. Functional studies using RNA interference (RNAi) of SJ genes in FCs result in defects in egg elongation, dorsal appendage morphogenesis, and BC migration. Together, these results reveal a strong similarity in the biogenesis of SJs between embryonic and follicular epithelia, demonstrate that at least some components of the SJ are required for morphogenesis in the ovary, and suggest that these roles may be independent of their role in forming an occluding junction.

\section{Materials and methods Fly stocks}

All Drosophila stocks were maintained on media consisting of corn meal, sugar, yeast, and agar on shelves at room temperature or in incubators maintained at a constant temperature of $25^{\circ} \mathrm{C}$. GAL4 lines used in this study are as follows: GR1-GAL4 [Bloomington Drosophila Stock Center (BDSC) \#36287], Slbo-GAL4, UAS-mCD8-GFP (BDSC\#76363), and C306-GAL4; GAL80 ${ }^{\text {ts }}$ /Cyo (a gift from Jocelyn McDonald, Kansas State University, Manhattan, KS, USA). RNAi stocks used for these studies are as follows: UAS-McrRNAi [BDSC\#65896 and Vienna Drosophila Resources Center (VDRC)\#100197], UAS-cora-RNAi (BDSC\#28933 and VDRC\#9787), UAS-Nrx-IV-RNAi (BDSC\#32424 and VDRC\#9039), UAS-Cont-RNAi (BDSC\#28923), UAS-mCherry-RNAi (BDSC\#35787), UAS-Lac-RNAi (BDSC\#28940), and UAS-sinu-RNAi (VDRC\#44929). UAS-Rab5 DN (BDSC\#9771) was used to inhibit normal Rab5 function and UASRab11-RNAi (BDSC\#27730) was used to knock down Rab11 in the FCs. UAS-GAL80 ${ }^{\text {ts }}$ (BDSC\#7108) was used to conditionally inhibit GR1-GAL4 activity in the UAS-Rab11-RNAi experiment. UAS-GFP (BDSC\#1521) was crossed to GR1-GAL4 as a control for the egg shape experiments. Slbo-GAL4, UAS-mCD8-GFP was crossed to UASmCherry-RNAi as a control for one set of BC migration studies, whereas C306-GAL4; GAL80 ${ }^{\text {ts }} /$ Cyo was crossed to UAS-Dcr (BDSC\#24646) as a control for the other set of BC migration studies. $w^{1118}$ (BDSC\# 5905) was used as the wild-type stock for determining the expression of Mcr, Cont, Nrx-IV, and Cora in the FCs.

\section{Fly staging}

$w^{1118}$ 1-2-day-old females and males were collected and reared at $25^{\circ} \mathrm{C}$ on fresh food sprinkled with yeast for 5-6 days before the females were dissection for antibody staining. For egg elongation analyses, crosses were maintained at $25^{\circ} \mathrm{C}$, and 1-2-day-old females (control and UAS-RNAi-expressing) were mated with sibling males and maintained at $29-30^{\circ} \mathrm{C}$ for 3 days before dissection. For BC migration analyses, Slbo-GAL4 crosses were kept at $25^{\circ} \mathrm{C}$, whereas C306-GAL4/UAS-Dcr; GAL80 $0^{\text {ts }} /$ SJ-RNAi crosses were kept at $18^{\circ} \mathrm{C}$ to prevent GAL4 activation. 1-2-day-old flies with the appropriate genotype (Slbo-GAL4, UAS-mCD8-GFP/UAS-RNAi, or C306-GAL4/UAS-Dcr; UAS-RNAi; GAL80 ${ }^{\text {ts }}$ ) were shifted to 29$30^{\circ} \mathrm{C}$ for 48 hours before dissection. It should be noted that by crossing UAS-GFP to C306-GAL4, we observed the expression of GFP in polar cells (PCs) in stage 10, but not stage 9 egg chambers (data not shown). For the Rab11-RNAi experiment, crosses were maintained at $18^{\circ} \mathrm{C}$ and 2-3-day-old males and females with the appropriate genotype (GR1-GAL4>UAS-mCherry-RNAi, UASGAL80ts or GR1-GAL4>UAS-Rab11-RNAi, UAS-GAL80ts) were collected and reared at $29-30^{\circ} \mathrm{C}$ overnight before dissection. For the Rab5 ${ }^{D N}$ experiment, crosses were maintained at $25^{\circ} \mathrm{C}$, and $1-2-$ day-old females were mated to sibling males and maintained at 29-30 ${ }^{\circ} \mathrm{C}$ for 3 days before dissection.

\section{Egg aspect ratio measurements}

Stage 14 egg chambers were selected for analysis based on the overall morphology of the egg and the absence of NCs nuclei by DAPI staining. Stage 14 egg chambers that have irregular edges or touch other egg chambers were excluded from the analysis to prevent inaccurate measurements. Egg length (anterior-posterior) and width (dorsal-ventral) were measured using the ImageJ/Fiji (http:// fiji.sc) (Schindelin et al. 2012) straight-line tool, and aspect ratio was calculated as length divided by width using Microsoft Excel.

\section{BC migration quantification}

Stage 10 egg chambers were identified based on the morphology of the egg (oocyte occupies half the egg chamber, whereas the other half is occupied by the NCs and centripetal cells). We used the GFP signal in Slbo-GAL4 crosses and DAPI and/or Fas3 staining in c306-GAL4 crosses to identify the location of the BC cluster in stage 10 egg chambers. The location of the BC cluster was quantified and grouped into four categories-complete, incomplete, failed migration, and disassociated cluster based on the location of the cluster relative to the oocyte in a stage 10 egg chamber (Figure 5). In some cases, BC clusters display two phenotypes such as complete and dissociated. In this case, we quantified both phenotypes in one egg chamber.

\section{Immunostaining and image acquisition}

Ovaries were dissected in $1 \mathrm{X}$ Phosphate-buffered saline (PBS), fixed in 4\% Paraformaldehyde for 20 minutes, washed three times in $1 \mathrm{X}$ PBS, and then permeabilized in a block solution (1X PBS + $0.1 \%$ Triton $+1 \%$ Normal Donkey Serum) for 30 minutes before incubation with primary antibodies either overnight at $4^{\circ} \mathrm{C}$ or 2-4hours at room temperature. The following antibodies were used at the given dilutions: guinea pig (gp) anti-Cont 1:2000 (Faivre-Sarrailh et al. 2004) and rabbit (rab) anti-Nrx-IV 1:500 (Baumgartner et al. 1996) obtained from Manzoor Bhat, University of Texas Health Science Center, San Antonio, TX, USA, gp antiMcr 1:1000 (Hall et al. 2014), mouse (m) anti-Cora [C566.9 and 
C615.16 mixed 1:1, obtained from the Developmental Studies Hybridoma Bank (DSHB) at the University of Iowa, Iowa City, IA, USA; Fehon et al. 1994] 1:50, rat anti-DE-cad (DCAD2, DSHB) 1:27, and $\mathrm{m}$ anti-Fas3 (7G10, DSHB) 1:260. DAPI (1 mg/ml) was used at a dilution of 1:1000. Cy2-, Cy3-, and Cy5-conjugated secondary antibodies were obtained from Jackson ImmunoResearch Laboratories (West Grove, Pennsylvania, PA, USA) and were used at 1:500

Images were acquired using an Olympus FV1000 confocal microscope equipped with Fluoview software (version 4.0.3.4). Objectives used included an UPLSAPO 20X Oil (NA: 0.85), a PLANAPO 60X Oil (NA: 1.42), and an UPLSAPO 100X Oil (NA: 1.40). Stage 14 egg chambers were imaged using Nikon Eclipse 80i compound microscope using a Nikon Plan Apo 10X air objective (NA: 0.45). Raw images were rotated and cropped in ImageJ/Fiji. Micrographs were adjusted for brightness using Adobe Photoshop 21.1.1 (San Jose, CA, USA) or ImageJ/Fiji. Adobe Illustrator 24.1 was used to compile the figures.

\section{Statistical analysis}

An unpaired t-test was used to calculate the P-values in egg chamber aspect ratio between control and SJ mutant stage 14 egg chambers using GraphPad Prism 8 (https://www.graphpad.com) (version 8.4.2).

\section{Data availability}

Fly stocks are available upon request. Supplementary files are available at figshare: https://doi.org/10.25387/g3.14387618. Supplementary Figure S1 shows the efficiency of RNAi knockdown in the FE of stage 12 egg chambers. Supplementary Figure S2 shows the range of dorsal appendage phenotypes found in GR1>SJ-RNAi stage 14 egg chambers. Supplementary Figure S3 shows the expression of Contactin during BC migration. Supplementary Figure S4 shows the expression of Nrx-IV during BC migration. Supplementary Figure S5 shows the expression of Coracle during BC migration. Supplementary Table S1 shows the raw length, width, and aspect ratios data for egg shape experiment shown in Figure 3, and the raw data for the BC migration studies presented in Figure 5. The authors affirm that all the data necessary for confirming the conclusions of the article are present within the article, figures, and supplemental files.

\section{Results}

\section{SJ proteins are expressed in FCs throughout oogenesis}

While a few SJ proteins have previously been reported to be expressed in the Drosophila ovary (Wei et al. 2004; Schneider et al. 2006; Hall et al. 2014; Maimon et al. 2014; Felix et al. 2015; Ben-Zvi and Volk 2019), a thorough analysis of their tissue distribution and subcellular localization throughout oogenesis is lacking. We therefore examined the spatial and temporal expression of four SJ proteins: Mcr, Cont, Nrx-IV, and Cora (Fehon et al. 1994; Baumgartner et al. 1996; Faivre-Sarrailh et al. 2004; Bätz et al. 2014; Hall et al. 2014). These four proteins are core components of the junction for which well-characterized antibodies are available.

At early stages of oogenesis (stages 2-8), Mcr, Cont, and Nrx-IV all localize at the lateral membrane of FCs and NCs, and also show a punctate distribution in these cells (Figure 1, D-F). Mcr, Cont, and Nrx-IV are also more strongly expressed in PCs than the surrounding FCs (asterisks in Figure 1, D-F). Cora is more uniformly localized along the lateral membrane of the FCs, including the PCs (Figure 1G and data not shown). These SJ proteins are additionally expressed in SCs (arrowheads in Figure 1, D and E and data not shown). Beginning at stage 10B, Mcr, Nrx-IV, Cont, and Cora are gradually enriched at the apical-lateral membrane of the FCs just basal to the AJ. This localization is complete by stage 11 (arrows in Figure 2, B, D, F, and H) and persists until the end of oogenesis (Figure 2, C, E, G, and I). The timing of this apical-lateral enrichment of Mcr, Cont, Nrx-IV, and Cora coincides with the maturation of the SJ in the FCs based upon ultrastructural analysis (Mahowald 1972; Müller 2000), and so we will refer to this region as the presumptive SJ.

\section{SJ proteins are required for egg elongation and dorsal appendage morphogenesis}

Given our findings that Mcr, Cont, Nrx-IV, and Cora are expressed in the FE throughout oogenesis, and our previous studies indicating a role for SJ proteins in morphogenesis, we wondered whether SJ proteins might be required for morphogenetic processes in the FE. The FE plays critical roles in shaping the egg chamber and producing the dorsal appendage, while a subset of FE cells participates in BC migration to form the micropyle (Montell 2003; Horne-Badovinac 2020). Because SJ mutant animals die during embryogenesis, we used the GAL4-UAS-RNAi system to knockdown the expression of SJ proteins in the FCs (Brand and Perrimon 1993). To knock down expression of SJ proteins throughout the majority of oogenesis, we used GR1-GAL4, which is expressed in the FCs from stages 4-14 of oogenesis (Gupta and Schüpbach 2003; Wittes and Schüpbach 2018). We examined SJ protein expression in late-stage egg chambers for $\mathrm{Mcr}^{-}$, cora-, and Nrx-IV-RNAi to demonstrate that the RNAi was efficiently knocking down protein expression in this tissue (Supplementary Figure S1). In all, we tested Bloomington Transgenic RNAi Project (TRiP) lines made against six different SJ genes (Cont, cora, Mcr, Lac, Nrx-IV, and sinu). To examine overall egg chamber shape, we dissected stage 14 egg chambers from females expressing SJ-RNAi under the control of GR1-GAL4, imaged them on a compound microscope, and determined the aspect ratio of the egg chambers using measurements of egg chamber length and width using ImageJ/Fiji. Control stage 14 egg chambers (GR1-GAL4>UAS-GFP) had a mean aspect ratio of 2.3 (Figure $3, \mathrm{~A}$ and J). In contrast, the aspect ratio of stage 14 egg chambers from all GR1-GAL4>SJ-RNAi is statistically significantly smaller than the aspect ratio of the control egg chambers (aspect ratios from 1.7 to 2.1, unpaired ttest $P<0.0001$ ). All SJ-RNAi stage 14 egg chambers are also significantly shorter (mean length from 391.9 to $466 \mathrm{~mm}$ ) than the control egg chamber (mean length of $487.6 \mathrm{~mm}$ ) (unpaired t-test $\mathrm{P}<0.0001$; Figure $3 \mathrm{H}$ ). Similarly, all (mean of width from 211.8 to $239.6 \mathrm{~mm}$ ) but Mcr-RNAi (BDSC) and Cont-RNAi are significantly wider than the control egg chamber (mean of width $208 \mathrm{~mm}$ ) (unpaired t-test $P<0.0001$; Figure $3 \mathrm{I}$ ). To confirm the specificity of these results we also tested VDRC RNAi lines directed against Mcr, cora, and Nrx-IV, and found similar effects on egg shape (unpaired t-test $P<0.0001$; Figure 3 ). The raw data for all of these analyses can be found in Supplementary Table S1.

Further examination of stage 14 SJ mutant egg chambers revealed defects in dorsal appendage morphology. Dorsal appendages are tubular respiratory structures that form from two populations of the dorsal FE known as floor and roof cells (Duhart et al. 2017). The primary phenotypes we observed in the SJ-RNAiexpressing egg chambers were missing dorsal appendages, or appendages that appeared to be short or broken (Figure 3L and Supplementary Figure S2). In addition, nearly all of the SJ-RNAiexpressing dorsal appendages that were present appeared to have a thinner stalk than found in control egg chambers 

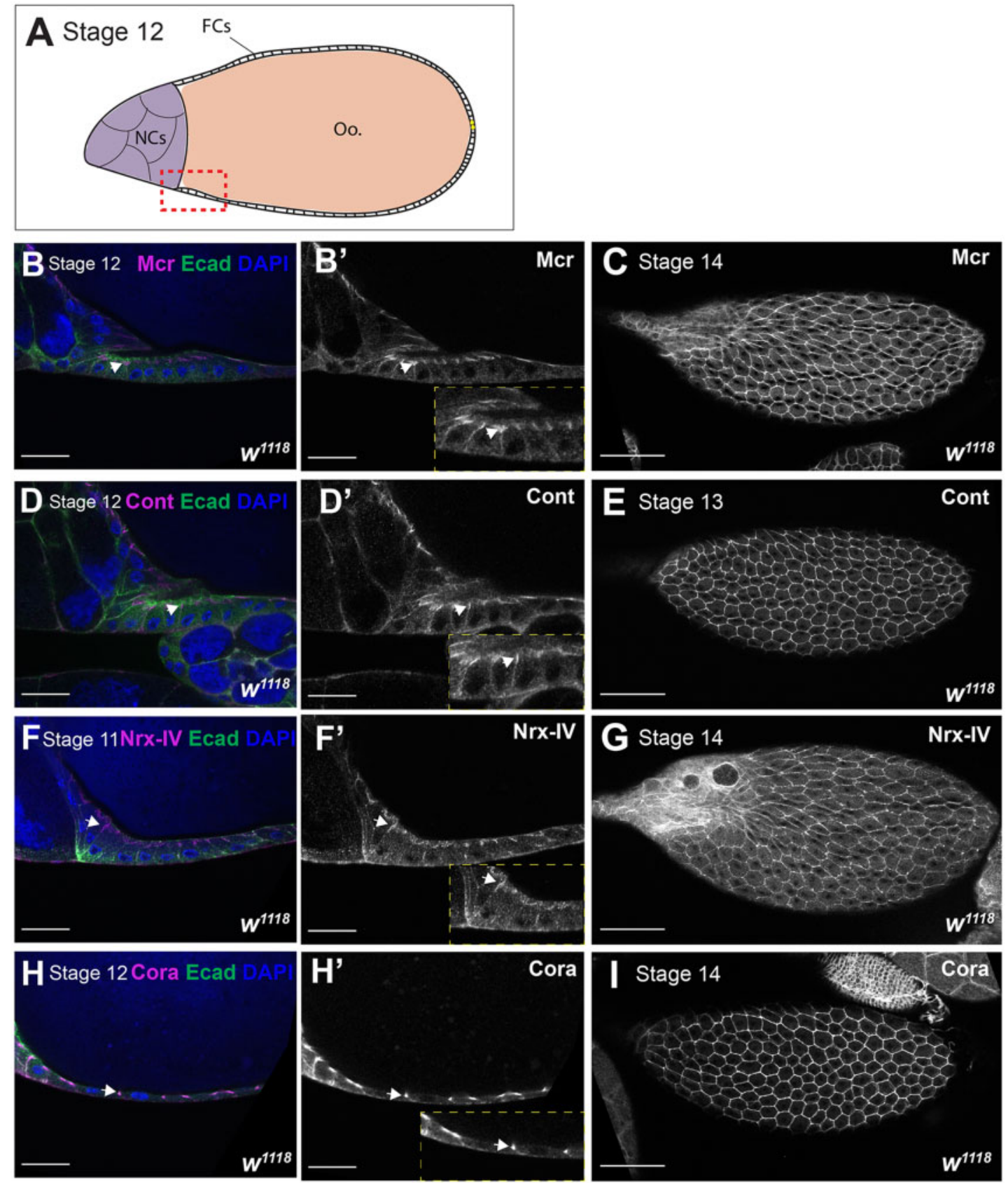

Figure $2 \mathrm{Mcr}$, Cont, Nrx-IV, and Cora localization at later stages of oogenesis. (A) Diagram of a stage 12 egg chamber. (B-I) Confocal optical sections of wild-type stages 11 and 12 egg chambers (B, D, F, and H) or stage 13 and14 egg chambers (C, E, G, and I) stained with antibodies against Mcr (B and C), Cont (D and E), Nrx-IV (F and G), or Cora (H and I) (magenta and in individual channel in B', D', F', and H') and co-stained with antibodies against Ecad (green) and labeled with DAPI (blue). The location of these sections overlaps the boundary between the oocyte (Oo.) and NCs and is depicted as the red box in the diagram shown in (A). Note that Mcr, Cont, Nrx-IV, and Cora become enriched at the apical-lateral region of FCs membrane (arrows in B, D, $\mathrm{F}$, and $\mathrm{H})$. Insets show higher magnification of the indicated areas. The expression of all of these proteins persists in stage 13/14 egg chambers (C, E, $G$ and I). Anterior is to the left. Scale bar $=25 \mu \mathrm{m}$ in B, D, F, and $\mathrm{H}$ and $100 \mu \mathrm{m}$ in C, E, G, and I.

(Supplementary Figure S2). In quantifying these phenotypes, both BDSC and VDRC RNAi lines against Mcr (BDSC: 52\%, VDRC: 18\%) and cora (BDSC: 15\% and VDRC: $21 \%$ ) produced egg chambers with defective dorsal appendages (Figure 3M). Similarly, 19\% of Cont-RNAi- and 13\% of Lac-RNAi-expressing egg chambers have either missing or broken dorsal appendages, however, the Nrx-IVRNAi BDSC line did not produce abnormal dorsal appendages, whereas 33\% of VDRC Nrx-IV-RNAi line resulted in defective dorsal appendages. We also did not observe defective dorsal appendages in sinu-RNAi-expressing egg chambers (Figure 3M).

\section{SJ proteins are expressed in polar and BC and are required for effective $B C$ migration}

The observation that Mcr, Cont, and Nrx-IV are strongly expressed in PCs and all FCs (Figure 1, D-F), motivated us to investigate their expression during the process of $B C$ migration. $B C$ migration occurs during stages 9-10 of oogenesis (Figure 4A). During stage 9 , signaling from the anterior PCs recruits a group of 4-8 adjacent FCs to form a cluster and delaminate apically into the egg chamber. The BC cluster is organized with a pair of PCs in 

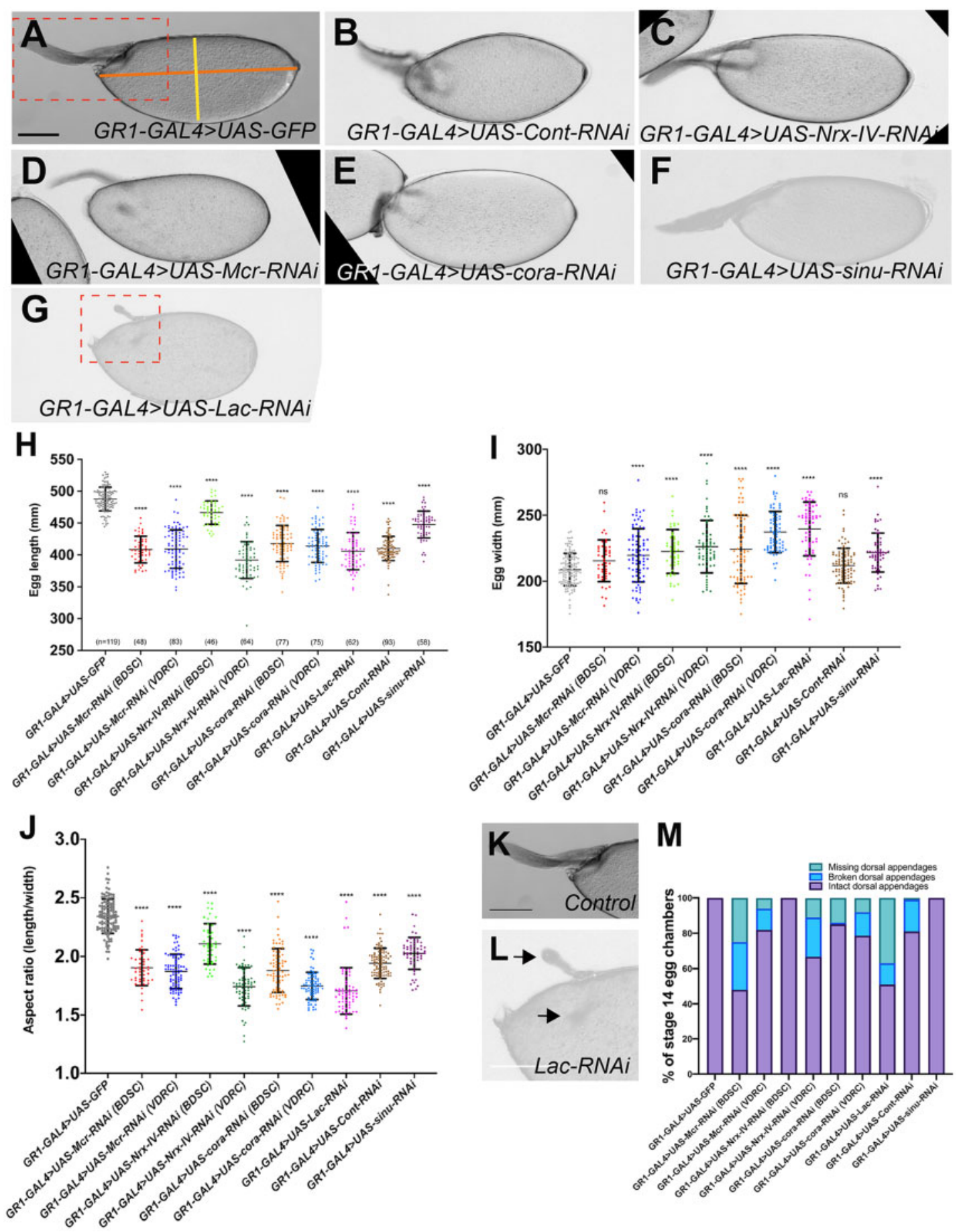

Figure 3 SJ genes are required for egg elongation. (A-G) Brightfield photomicrographs of stage 14 egg chambers. (A) GR1-GAL4>UAS-GFP, (B) GR1GAL4>UAS-Cont-RNAi, (C) GR1-GAL4>UAS-Nrx-IV-RNAi, (D) GR1-GAL4>UAS-Mcr-RNAi, (E) GR1-GAL4>UAS-cora-RNAi, (F) GR1-GAL4>UAS-sinu-RNAi, and (G) GR1-GAL4>UAS-Lac-RNAi. Images in this figure represent average phenotypes observed in each genotype. (H and I) Quantification of length and width of stage 14 egg chambers from control and SJ-RNAi egg chambers. (J) Quantification of the aspect ratio of length (orange line in A) to width (yellow line in A) from control and SJ-RNAi stage 14 egg chambers. Note that the aspect ratio of all SJ-RNAi expressing egg chambers are statistically significantly different from the control egg chambers (unpaired t-test; $P<0.0001$ ). (K and L) Zoomed images of GR1-GAL4>UAS-GFP (K) and GR1GAL4>UAS-Lac-RNAi (L) stage 14 egg chambers (from A and G) showing the dorsal appendages. The dorsal appendages in the Lac-RNAi egg chamber are either deformed or absent (arrows) compared to control dorsal appendages. (M) Quantification of dorsal appendage defects from control and SJ-RNAi stage 14 egg chambers. $n$, total number of egg chambers measured per genotype. Data represent individual values with mean \pm s.d. $P$-value $<0.0001$. Anterior is to the left. Scale bar $=100 \mu \mathrm{m}$.

the center surrounded by BCs. This cluster of cells migrates between the NCs until they reach the anterior side of the oocyte (Figure 4A). This process takes approximately 4-6hours and is complete in wild-type egg chambers by stage 10 of oogenesis
(Prasad and Montell 2007). The BC cluster, along with the migratory centripetal cells, collaborate to form the micropyle, a hole through which the sperm enters the egg (Montell 2003; HorneBadovinac 2020). Previous studies showed that Cora and Nrg are 

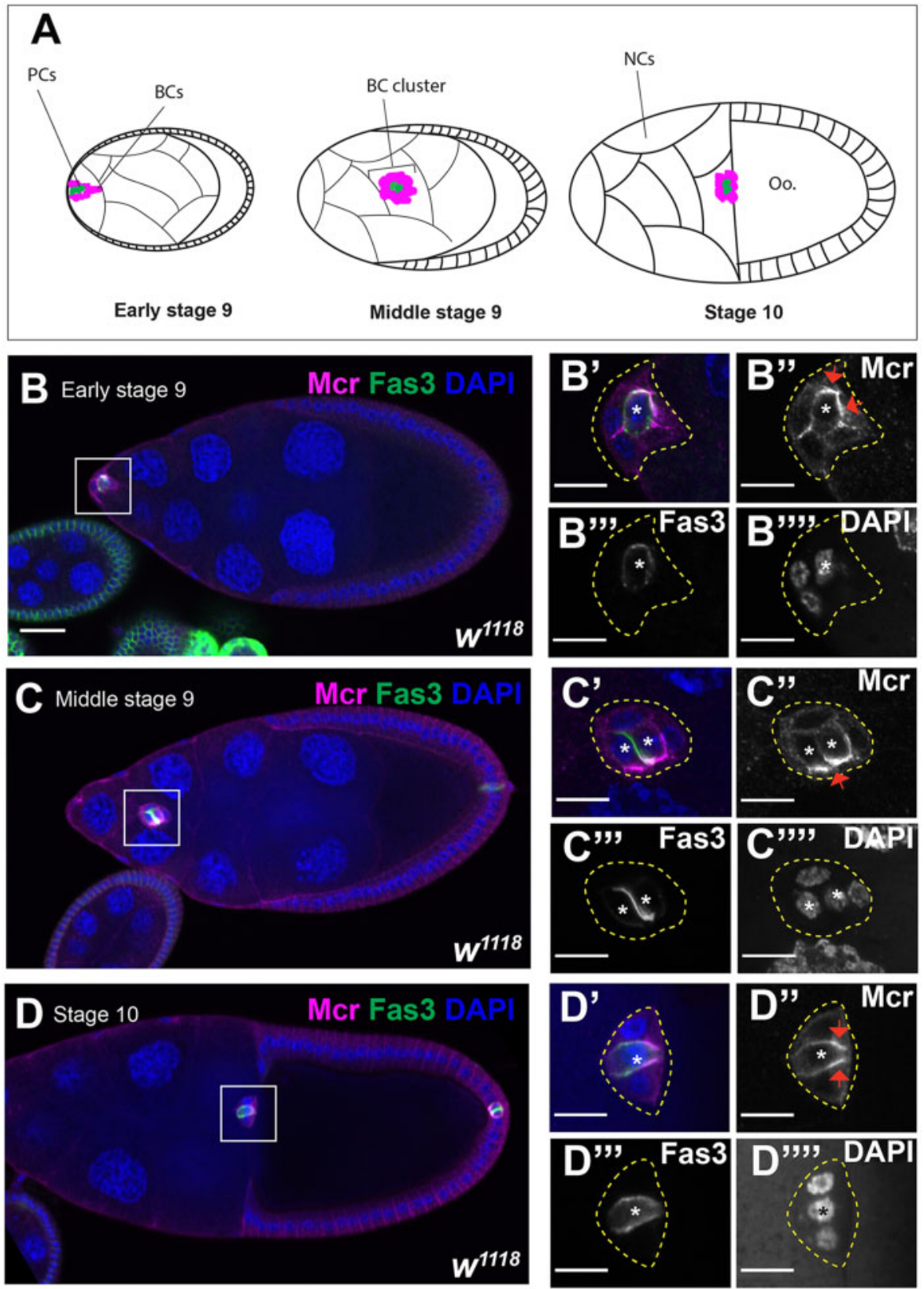

Figure $4 \mathrm{Mcr}$ expression during BC migration. (A) Diagram of BC migration. At early stage 9 of oogenesis, a group of 4-6 FCs are specified by the PCs (green) to become BCs (magenta). The BC/PC cluster delaminates apically and migrates between the NCs until it reaches the oocyte (Oo.) by stage 10 of oogenesis. (B-D) Confocal optical sections of wild-type stages 9 and 10 egg chambers stained with antibodies against Mcr (magenta, and in " panels), Fas3 (green, and in "' panels), and labeled with DAPI (blue, and in "' panels). PCs are indicated with asterisks. Mcr is expressed in the PCs and BCs with higher expression at the interface between the PCs and BCs. Note that Mcr appears to be most enriched along the boundary with BCs at the leading edge of the BC cluster (red arrows in B"-D"). Anterior is to the left. Scale bar = $25 \mu \mathrm{m}$ in (B-D) and $10 \mu \mathrm{m}$ in (B'-B"' and D'-D"').

expressed in the PCs during their migration (Wei et al. 2004; Felix et al. 2015). To determine if other SJ proteins are also expressed during BC migration, we stained stages 9 and 10 wild-type egg chambers with antibodies against Mcr, Cont, Nrx-IV, and Cora. To mark the PCs, we co-stained the egg chambers with Fasciclin 3 (Fas3; Snow et al. 1989; Khammari et al. 2011). Mcr, Cont, Nrx-IV, and Cora are all expressed in BC clusters throughout their migration (Figure 4, B-D and Supplementary Figures S3-S5). Interestingly, the expression of these SJ proteins in PCs appears highest at the interfaces between polar and BCs (Figure 4, B-D and Supplementary Figures S3-S5). SJ protein expression is also asymmetric in the BC cluster, with higher expression along BCs closest to the oocyte, raising the possibility that these proteins may respond to or direct leading-edge polarity in the migrating BC cluster (red arrows in Figure 4, B-D and Supplementary Figures S3-S5).

Given that Mcr, Cont, Nrx-IV, and Cora are expressed in BC clusters throughout BC migration, we wondered if they are also required for some aspect of this process. To address this issue, we used Slbo-GAL4 (Ogienko et al. 2018) to express RNAi against individual SJ genes specifically in BCs and analyzed the BC clusters at stage 10 in these ovaries. We noticed a range of defective migration phenotypes in these egg chambers and classified them into three nonexclusive groups: failed, incomplete, and 

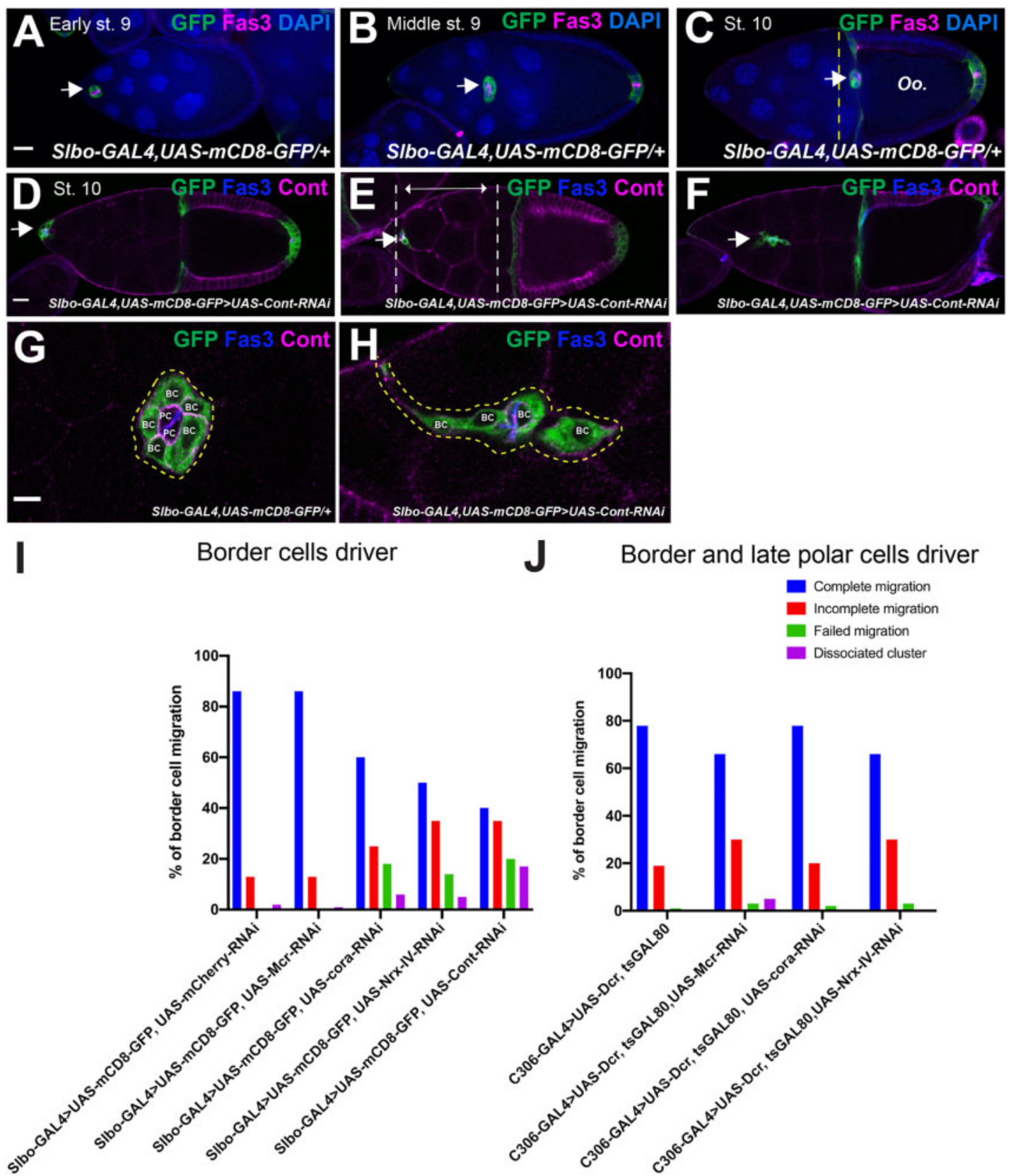

Figure $5 \mathrm{Mcr}$, Cont, Nrx-IV, and Cora are required for effective BC migration. (A-C) BC migration in control egg chambers. Egg chambers are immunostained with anti-Fas3 (magenta) to mark the PCs, GFP (green) to mark BCs, and labeled with DAPI (blue). Arrows indicate BC clusters. Note that in control egg chambers the BC cluster reaches the oocyte at stage 10 (C). (D-F) Stage 10 egg chambers expressing Cont-RNAi in BCs immunostained with anti-Cont (magenta) and anti-Fas3 (blue) showing examples of BC cluster migration defects: failed (arrow in D), incomplete (arrow in E), and incomplete with a disassociated BC cluster (arrow in F). Higher magnification of control (G) and Cont-RNAi-expressing (H) BC clusters showing the dissociation of a BC cluster observed in SJ-RNAi clusters. (I and J) Quantification of BC cluster phenotypes at stage 10 egg chambers in control and SJRNAi driven by either Slbo-GAL4 (I) or C306-GAL4 (J). Anterior is to the left. Scale bar $=25 \mu \mathrm{m}$.

dissociated clusters. Complete migration (Figure 5, A-C) is characterized by having the entire BC cluster physically touching or just adjacent the oocyte by the end of stage 10 (Figure 5C). A failed cluster is characterized by a BC cluster that has not delaminated from the FE by stage 10 (Figure 5D). An incomplete migration phenotype is characterized as an intact cluster that has not reached the oocyte by the end of stage 10 (Figure 5E, where the two dashed lines indicate the range of distances at which BC clusters were categorized as incomplete). Finally, a dissociated cluster phenotype is characterized by a cluster that has broken into a linear string of BCs or that has one or more BCs that remain between NCs and are not connected to the larger BC cluster (Figure 5, F and H).

In control stage 10 egg chambers (Slbo-GAL4; UAS-mCD8-GFP/ UAS-mCherry-RNAi), $86 \%(n=81)$ of BC clusters completed their migration, with the remainder showing incomplete migration (Figure 5I). In contrast, stage 10 egg chambers expressing RNAi against cora, Nrx-IV, or Cont in the BCs resulted in 58\% $(n=67)$, $50 \%(n=74)$, and $40 \%(n=85)$ of BC clusters completing migration, respectively (Figure 5I). The remaining cora-RNAi- and NrxIV-RNAi-BC clusters showed a combination of incomplete migration or failed to delaminate (Figure 5I). Cont-RNAi-BC clusters also 
showed $35 \%$ of incomplete BC migration, but additionally had $17 \%$ of the clusters disassociating during their migration (Figure 5, E-I). Mcr-RNAi-BC clusters were indistinguishable from controls with $86 \%(n=94)$ completing their migration and the remainder showing only incomplete migration (Figure 5I).

To extend these studies, we examined BC migration in egg chambers expressing RNAi against SJ genes using the C306-GAL4 driver. C306-GAL4 is expressed in the BCs throughout the process of BC migration (Murphy and Montell 1996) and in PCs just at stage 10 (H.A., unpublished observation). In control egg chambers (C306-GAL4/UAS-Dcr; GAL80 ${ }^{\text {ts }} /+$ ), 78\% ( $n=91$ ) of BC clusters completed their migration, and 19\% displayed incomplete migration (Figure 5J). Stage 10 egg chambers expressing C306>Mcr-RNAi displayed $66 \%(n=98)$ complete BC migration with $30 \%$ showing incomplete migration, 5\% showing dissociated clusters, and 3\% showing failed BC migration (Figure 5J). Similarly, egg chambers expressing C306>Nrx-IV-RNAi displayed 66\% $(n=59)$ complete BC migration with 30\% showing incomplete migration and 3\% failing to delaminate (Figure $5 \mathrm{~J})$. Finally, $78 \%(n=70)$ of stage 10 C306>cora-RNAi-expressing BCs displayed complete BC migration, whereas $20 \%$ showed incomplete migration and $3 \%$ failed to delaminate (Figure 5J).

\section{SJ biogenesis in the follicular epithelium}

The redistribution of SJ proteins in the FCs of later stage egg chambers resembles the dynamic relocalization of SJ proteins during the biogenesis of the junction during embryogenesis (Tiklová et al. 2010). In embryonic epithelia, SJ protein enrichment at the apical-lateral domain (presumptive SJ) requires endocytosis and recycling of SJ proteins to the membrane, and is interdependent on the presence of all core SJ proteins (Ward et al. 1998; Hall et al. 2014). Coincident with the strong localization of SJ proteins to the presumptive SJ at stage 16 of embryogenesis, ladderlike electron-dense intermembrane septa are visible by electron microscopy that becomes progressively organized by stage 17 (Schulte et al. 2003; Hildebrandt et al. 2015). We therefore sought to determine if similar processes occur during the formation of SJs in the FE.

To test for the interdependence of SJ proteins for localization, we examined Cora and Mcr expression in wild- type, Mcr-RNAi, and Nrx-IV-RNAi stage 12 FCs. In wild-type stage 12 egg chambers, Cora is strongly co-localized with Mcr at the apical-lateral domain of the FCs (completely penetrant in 95 cells from 31 egg chambers) (Figure 6A). In contrast, Cora and Mcr are mislocalized along the lateral domain in stage 12 Nrx-IV-RNAi FCs (Figure 6B), much like they are in stage 2-8 wild-type FCs (Figure 1D). Specifically, 6 out of 20 Nrx-IV-RNAi FCs cells from 19 egg chambers showed complete mislocalization, whereas 13 of these 20 cells showed largely lateral localization with some degree of apical enrichment. Similarly, in stage 12 Mcr-RNAi FCs, Cora was mislocalized along the lateral membrane in 39 out of 47 cells examined from 19 egg chambers, with the remaining 8 cells showing some enrichment of Cora at the apical lateral domain (Figure 6C). Notably, cells that showed apical enrichment of Cora also retained small foci of Mcr expression that overlaps with the enriched Cora (Figure 6D), suggesting the perdurance of $\mathrm{Mcr}$ in these cells may have allowed for normal SJ organization. Together, these experiments indicate that SJ biogenesis in the FE of late-stage egg chambers requires the expression of at least some core SJ proteins.

We next wanted to investigate whether the relocalization of SJ proteins to the presumptive SJ required endocytosis and recycling. In the embryonic hindgut, Cora, Gliotactin (Gli), Sinu, and Melanotransferrin (Mtf) localize with the early endosomal marker, Rab5, and partially localize with the recycling marker, Rab11 during SJ biogenesis (Tiklová et al. 2010). Moreover, blocking Rab5 or Rab11 function prevents Cora, Gli, Sinu, and Mtf apical-lateral localization (Tiklová et al. 2010), and thus SJ formation.
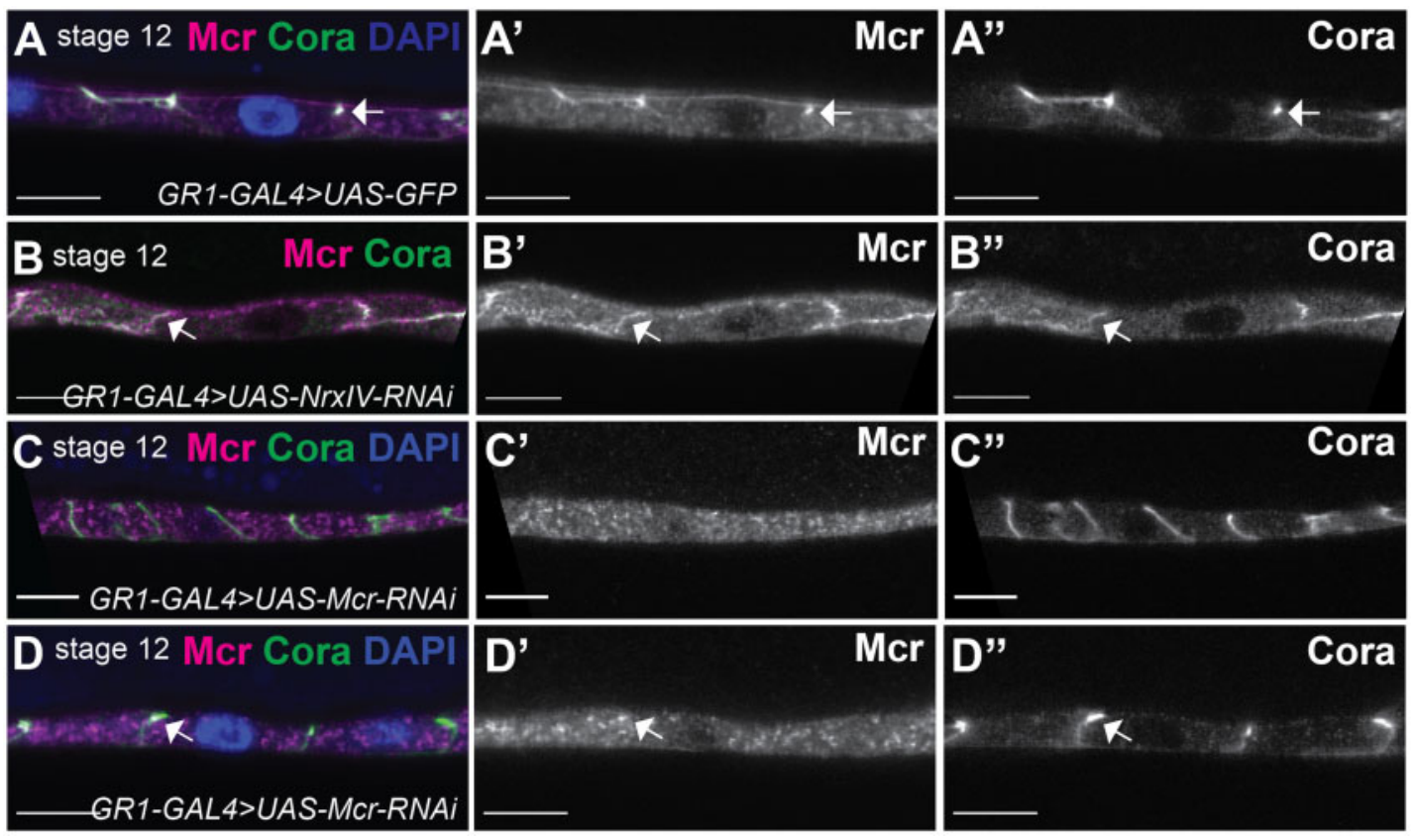

Figure 6 The apical-lateral localization of Cora depends on Mcr and NrxIV. (A-D) Confocal optical sections of stage 12 FCs stained with antibodies against Mcr (magenta, and in ' panels) and Cora (green, and in " panels) and labeled with DAPI (blue). Mcr and Cora co-localize at the presumptive SJ in control stage 12 egg chambers (A), whereas Mcr and Cora localize along the lateral membrane of NrxIV-RNAi expressing FCs (B). In most Mcr-RNAiexpressing stage 12 egg chambers, Cora localizes laterally (C), whereas in some egg chambers, Cora is enriched apically (arrow in D"), but is often associated with Mcr puncta (arrow in D'). Scale bar $=10 \mu \mathrm{m}$. 

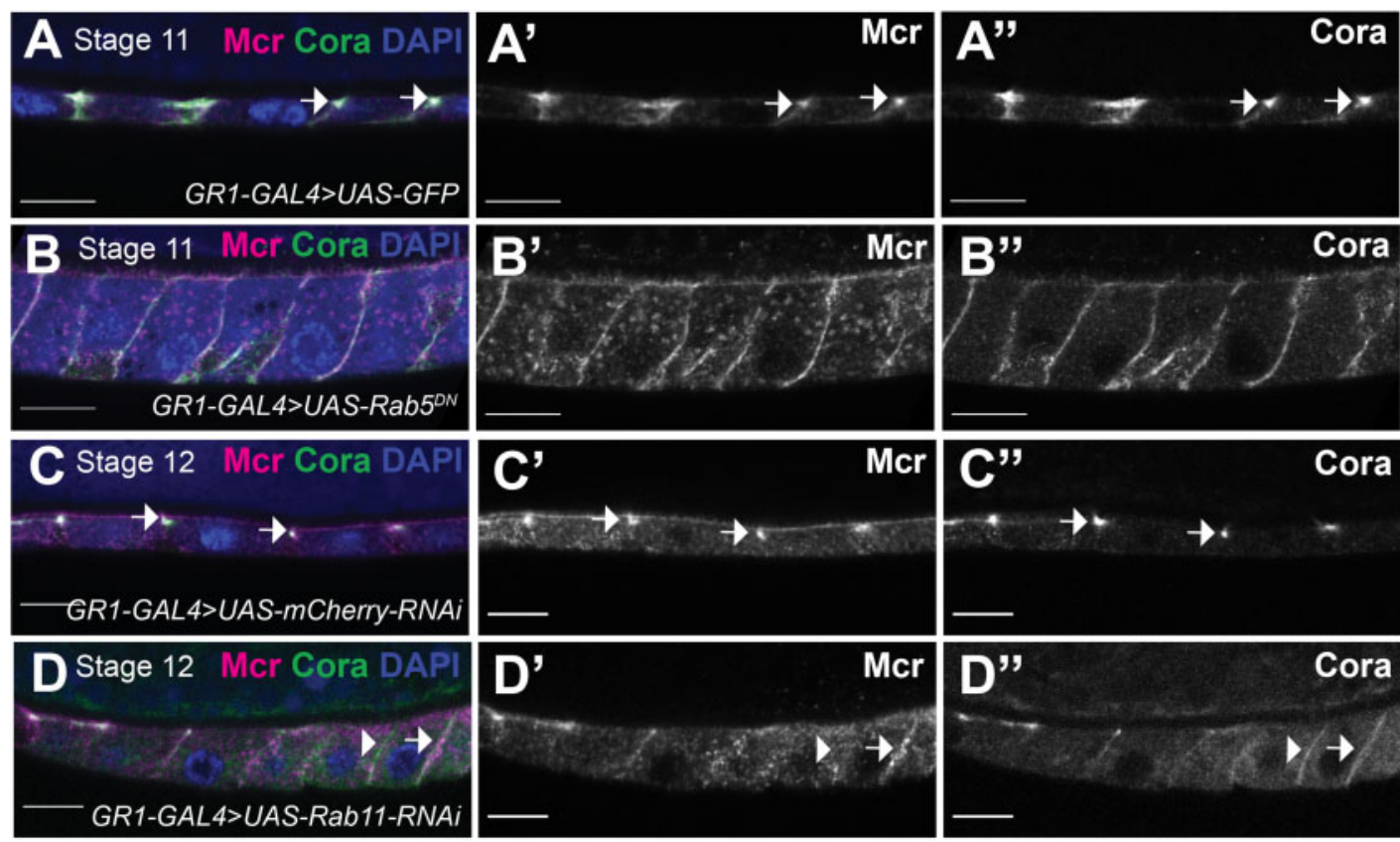

Figure $7 \mathrm{Mcr}$ and Cora require Rab5 and Rab11 for their correct localization at the SJ. (A-D) Confocal optical sections of stage 11 (A and B) or stage 12 (C and D) FCs stained with antibodies against Mcr (magenta, and in ' panels) and Cora (green, and in " panels) and labeled with DAPI (blue). While Mcr and Cora co-localize at the apical-lateral membrane of stages 11 and 12 control FCs (arrows in A and C), both Cora and Mcr fail to localize at the SJ in Rab5 $5^{\mathrm{DN}}$-expressing FCs (B). In Rab11-RNAi-expressingFCs (D), Cora localizes along the lateral membrane (arrowhead in D"), whereas Mcr is either missing (arrowhead in D') or localizes along the lateral membrane (arrow in D'). Anterior is to the left. Scale bars $=10 \mu \mathrm{m}$.

To determine if similar processes occur during SJ formation in FCs, we expressed a dominant negative version of Rab5 (UASRab5 ${ }^{\mathrm{DN}}$ ) in FCs using GR1-GAL4 and examined the expression of $\mathrm{Mcr}$ and Cora in stage 11 FCs. In wild-type FCs at that stage, Mcr and Cora are enriched at the apical-lateral membrane (completely penetrant in 91 cells from 15 egg chambers; arrows in Figure 7A), whereas both $\mathrm{Mcr}$ and Cora remains localized along the lateral membrane in the Rab5 $5^{\mathrm{DN}}$-expressing FCs (completely penetrant in 97 cells from 15 egg chambers; Figure 7B). Similarly, Cora and $\mathrm{Mcr}$ co-localize at the apical-lateral membrane of the FCs of stage 12 egg chambers (completely penetrant in 64 cells from 15 egg chambers; arrows in Figure 7C), whereas knocking down the expression of Rab11 in stage 12 FCs (via GR1>Rab11RNAi) resulted in the mislocalization of Cora and Mcr in 44 cells out of 70 from 16 egg chambers. In 23 of these 44 cells both Mcr and Cora are mislocalized along the entire lateral membrane (arrow in Figure 7D), whereas in the other 21 cells, Cora is mislocalized along the lateral membrane, while $\mathrm{Mcr}$ is in cytoplasmic puncta or completely missing (arrowhead in Figure 7D). Interestingly, we noted that the FE in Rab5 $5^{\mathrm{DN}}$ - and Rab11-RNAiexpressing egg chambers are taller in the apical/basal dimension than similarly staged wild-type egg chambers (compare Figure 7A with Figure 7B and Figure 7C with Figure 7D), although the effect is greater with $\mathrm{Rab5}^{\mathrm{DN}}$ than with Rab11-RNAi. Taken together, these results indicate that similar to embryonic epithelia, the maturation of SJs in the FE requires Rab5-mediated endocytosis and Rab11-mediated recycling. in

\section{Discussion}

In this study, we have demonstrated that a subset of SJ proteins is expressed in egg chambers throughout oogenesis and are required for critical morphogenetic processes that shape the egg, including egg chamber elongation, dorsal appendage formation, and BC migration. Interestingly, the subcellular localization of these SJ proteins in the ovarian FCs changes coincident with the establishment of the occluding junction in much the same way that they do during embryogenesis in ectodermal epithelial cells (Tiklová et al. 2010), suggesting that a similar maturation process for the SJ occurs in this tissue.

\section{Biogenesis of the $\mathrm{SJ}$ in the $\mathrm{FE}$}

The mechanisms of SJ biogenesis in embryonic epithelia has been well-studied and involves several steps in which transmembrane SJ proteins are first localized all along lateral plasma membranes (by stage 12 of embryogenesis), but then must be endocytosed and recycled back to the plasma membrane prior to aggregating in the region of the presumptive SJ (between stages 13 and 16; Tiklová et al. 2010). Prior to this relocalization step, SJ proteins show high mobility in the plane of the membrane by Fluorescence Recovery After Photobleaching (FRAP) analysis, but become strongly immobile as the relocalization is occurring (Oshima and Fehon 2011). As these steps are occurring (e.g., stage 14 of embryogenesis), electron-dense material begins to accumulate between adjacent cells in the presumptive SJ that takes on the appearance of ladder-like septa by stage 17 of embryogenesis (Tepass and Hartenstein 1994). Functional studies indicate that the occluding function of the junction is established late in embryogenesis, near the end of stage 15 (Paul et al. 2003). Importantly, the process of SJ biogenesis is interdependent on the function of all core components of the junction and several accessory proteins including Rab 5 and Rab 11 (Ward et al. 1998; Genova and Fehon 2003; Tiklová et al. 2010).

Here, we observe that many of these same steps occur in the maturation of SJs in the FE. We first show that SJ proteins are expressed in the FE beginning early in ovarian development where they localize all along the lateral membrane (Figure 1). These proteins appear to become enriched at the presumptive SJ 
by stage 11 (Figure 6). The relocalization of SJ proteins to the SJ requires core SJ proteins including Mcr and Nrx-IV, and accessory proteins including Rab5 and Rab11 (Figures 6 and 7). Prior studies indicate the presence of electron dense material between FE cells as early as stage 6 (Müller 2000), with a ladder-like appearance of extracellular septa by stage 10B (Mahowald 1972). A recent study of the occluding function in the FE shows a similar pattern of protein localization for endogenously tagged Neuroglian-YFP and Lachesin-GFP, and demonstrates that an intact occluding junction is formed during stage 11 of oogenesis (Isasti-Sanchez et al. 2021). It is interesting to note the FE is a secondary epithelium initiated by a mesenchymal to epithelial transition (Tepass et al. 2001), and yet SJ biogenesis appears to be very similar to that observed in the primary epithelia found in the embryo.

\section{SJ proteins are required for morphogenetic events during oogenesis}

The similarities in the dynamic expression of SJ proteins in the FE and embryonic epithelia, coupled with the observation that SJ proteins are required for numerous embryonic developmental events (Hall et al. 2014, and references therein) motivated us to explore whether SJ proteins have similar roles in morphogenetic processes that shape the egg. Using a targeted RNAi approach, we show that reducing the expression of Mcr, Nrx-IV, Cont, cora, sinu, or Lac throughout oogenesis in the FCs results in a significantly smaller aspect ratio of stage 14 egg chambers, with many showing additional defects in dorsal appendages (Figure 3 and Supplementary Figure S2). The initiation and maintenance of egg elongation are achieved at various stages throughout oogenesis (Bilder and Haigo 2012; Gates 2012; Cetera and Horne-Badovinac 2015). From stages 3-6, a gradient of JAK-STAT signaling is required at each pole of the egg chamber to promote Myosin IIbased apical cell contractions (Alégot et al. 2018). From stages 68 , collective FC rotation over the germline generates a robust planar polarized molecular corset consisting of basement membrane protein fibrils aligned with the basal actin cytoskeleton that is required for egg elongation (Gutzeit et al. 1991; Bateman et al. 2001; Frydman and Spradling 2001; Viktorinová et al. 2009; Haigo and Bilder 2011; Horne-Badovinac et al. 2012; Cetera et al. 2014; Isabella and Horne-Badovinac 2016; Cerqueira Campos et al. 2020). During the middle stages of oogenesis (stages 9 and 10), basal actin stress fibers undergo actomyosin contractions, which contribute to egg elongation (He et al. 2010; Qin et al. 2017). Finally, later in oogenesis, the maintenance of a planar polarized molecular corset is required to retain an elongated egg chamber shape (Haigo and Bilder 2011; Cha et al. 2017; Cerqueira Campos et al. 2020). Future studies are needed to determine how SJ proteins participate in these events to establish and/or maintain egg shape. Because many of the events involved in egg elongation occur prior to the formation of the occluding junction in the FE, it raises the possibility that much like during embryogenesis, SJ proteins may have a function in morphogenesis that is independent of their role in forming a tight occluding junction.

Stage 14 egg chambers from many of the SJ-RNAi lines possessed aberrant dorsal appendages, often characterized by misshapen, broken, or missing appendages (Figure 3 and Supplementary Figure S2). The formation of dorsal appendages occurs during stages 10B-14 and requires cell shape changes and cell rearrangements, coupled with chorion protein secretion (Dorman et al. 2004). Similar morphogenetic processes are required for dorsal closure and head involution during embryogenesis (VanHook and Letsou 2008; Hayes and Solon 2017), defects strongly associated with zygotic loss of SJ expression in the embryo (Hall and Ward 2016). We are interested to determine if the mechanism by which SJ proteins contribute to dorsal appendages formation and dorsal closure and head involution are similar. Potential roles could involve regulating the cytoskeleton to facilitate cell shape changes and rearrangements. These observations also suggest the possibility that SJ proteins may be required for chorion protein secretion or crosslinking. Broken and missing dorsal appendages may result from mechanical disruption due to chorion defects. We also noticed mature SJ-RNAi eggs with a thin chorion (data not shown). Notably, embryos with mutations in several different SJ genes show defects in the embryonic cuticle including faint denticle belts and delamination of cuticle layers (Lamb et al. 1998; Hall and Ward 2016).

Our observation that specifically knocking down the expression of several SJ proteins in BCs results in defective BC migration (Figure 5) supports a role for SJ proteins in morphogenesis, independent of their role in forming an occluding junction. The phenotypes we observed include failing to complete migration and partial disassociation of the complex by the end of stage 10, which is prior to the formation of an intact SJ. Although the penetrance of these phenotypes is mild (Figure 5, I and J), it is possible that these phenotypes underestimate the full requirement of $\mathrm{SJ}$ proteins in BC migration since this process takes a relatively short time (4-6hours) (Prasad and Montell 2007), while SJ proteins are thought to be very stable in the membrane (Oshima and Fehon 2011). One caveat to the idea that perdurance may account for the mild phenotypes is that C306-GAL4 does not appear to produce a stronger phenotype than slbo-GAL4, even though it is expressed earlier and is presumably knocking down SJ proteins longer. Perhaps knocking the proteins down more quickly using the DeGradFP system (Caussinus et al. 2021) could address this possibility in the future. These phenotypes also indicate a potential role for SJ proteins in cell adhesion and/or cell polarity during migration. Specifically, we note that Mcr appears to be enriched in PC membranes that contact BCs at the leading edge of the cluster (those that are oriented closest to the oocyte) in wild-type egg chambers (Figure 4). Whether SJ proteins are required for aspects of planar polarity in the BC cluster is an interesting unanswered question. Perhaps the incomplete migration defect results from a meandering migration through the NCs, something that has been observed for knockdown of DE-Cadherin in BCs (Cai et al. 2014). Live imaging studies should be able to distinguish between pathfinding defects and a general reduction in speed or premature stopping. A role for SJ proteins in cell adhesion in the ovary has been reported previously. Reducing the level of Nrg in FCs results in the failure of FCs that are born outside of the FE due to misoriented spindles to reintegrate into the FE, indicating a role for $\mathrm{Nrg}$ in lateral cell adhesion (Bergstralh et al. 2015). Similarly, expressing a null allele of Nrg in FCs enhances the invasive tumor phenotype of a Discs Large (Dlg) mutation (Wei et al. 2004).

\section{Conflicts of interest}

None declared.

\section{Acknowledgments}

The authors thank Jocelyn McDonald, the Bloomington Drosophila Stock Center, and the Vienna Drosophila RNAi Center for fly stocks. They thank Manzoor Bhat and the Developmental Studies Hybridoma Bank (created by the NICHD of the NIH and maintained at The University of Iowa, Department of Biology, Iowa City, IA 52242, USA) for antibodies used in this study. They 
thank Brian Ackley for the use of his Olympus FV1000 confocal microscope. They thank Lindsay Ussher for preliminary studies on BC migration and thoughtful discussions on the project. They also thank Sally Horne-Badovinac, Jocelyn McDonald, Yujun Chen, and members of the Ward lab for helpful discussion about the project and manuscript. This project was supported by a grant from the National Science Foundation (IOS 2111069) to REW.

\section{Literature cited}

Alégot H, Pouchin P, Bardot O, Mirouse V. 2018. Jak-Stat pathway induces Drosophila follicle elongation by a gradient of apical contractility. ELife. 7:e32943.

Banerjee S, Bainton RJ, Mayer N, Beckstead R, Bhat MA. 2008. Septate junctions are required for ommatidial integrity and blood-eye barrier function in Drosophila. Dev Biol. 317:585-599.

Bateman J, Reddy RS, Saito H, Van Vactor D. 2001. The receptor tyrosine phosphatase Dlar and integrins organize actin filaments in the Drosophila follicular epithelium. Curr Biol. 11:1317-1327.

Bätz T, Förster D, Luschnig S. 2014. The transmembrane protein macroglobulin complement-related is essential for septate junction formation and epithelial barrier function in Drosophila. Development. 141:899-908.

Baumgartner S, Littleton JT, Broadie K, Bhat MA, Harbecke R, et al. 1996. A Drosophila neurexin is required for septate junction and blood-nerve barrier formation and function. Cell. 87:1059-1068.

Behr M, Riedel D, Schuh R. 2003. The claudin-like megatrachea is essential in septate junctions for the epithelial barrier function in Drosophila. Dev Cell. 5:611-620.

Ben-Zvi DS, Volk T. 2019. Escort cell encapsulation of Drosophila germline cells is maintained by irre cell recognition module proteins. Biol Open. 8:bio039842.

Bergstralh DT, Lovegrove HE, St Johnston D. 2015. Lateral adhesion drives reintegration of misplaced cells into epithelial monolayers. Nat. Cell Biol. 17:1497-1503.

Bilder D, Haigo SL. 2012. Expanding the morphogenetic repertoire: perspectives from the Drosophila egg. Dev Cell. 22:12-23.

Brand AH, Perrimon N. 1993. Targeted gene expression as a means of altering cell fates and generating dominant phenotypes. Development. 118:401-415.

Cai D, Chen SC, Prasad M, He L, Wang X, et al. 2014. Mechanical feedback through E-cadherin promotes direction sensing during collective cell migration. Cell. 157:1146-1159.

Caussinus E, Kanca O, Affolter M. 2021. Fluorescent fusion protein knockout mediated by anti-GFP nanobody. Nat Struct Mol Biol. 19:117-121.

Cerqueira Campos FC, Dennis C, Alégot H, Fritsch C, Isabella A, et al. 2020. Oriented basement membrane fibrils provide a memory for F-actin planar polarization via the dystrophin-dystroglycan complex during tissue elongation. Development. 147:dev186957.

Cetera M, Horne-Badovinac S. 2015. Round and round gets you somewhere: collective cell migration and planar polarity in elongating Drosophila egg chambers. Curr Opin Genet Dev. 32:10-15.

Cetera M, Ramirez-San Juan GR, Oakes PW, Lewellyn L, Fairchild MJ, et al. 2014. Epithelial rotation promotes the global alignment of contractile actin bundles during Drosophila egg chamber elongation. Nat Commun. 5:5511

Cha IJ, Lee JH, Cho KS, Lee SB. 2017. Drosophila tensin plays an essential role in cell migration and planar polarity formation during oogenesis by mediating integrin-dependent extracellular signals to actin organization. Biochem Biophys Res Commun. 484:702-709.

Dorman JB, James KE, Fraser SE, Kiehart DP, Berg CA. 2004. Bullwinkle is required for epithelial morphogenesis during Drosophila oogenesis. Dev Biol. 267:320-341.

Duhart JC, Parsons TT, Raftery LA. 2017. The repertoire of epithelial morphogenesis on display: progressive elaboration of Drosophila egg structure. Mech Dev. 148:18-39.

Faivre-Sarrailh C, Banerjee S, Li J, Hortsch M, Monique L, et al. 2004. Drosophila contactin, a homolog of vertebrate contactin, is required for septate junction organization and paracellular barrier function. Development. 131:4931-4942.

Fehon RG, Dawson IA, Artavanis-Tsakonas S. 1994. A Drosophila homologue of membrane-skeleton protein 4.1 is associated with septate junctions and is encoded by the coracle gene. Development. 120:545-557.

Felix M, Chayengia M, Ghosh R, Sharma A, Prasad M. 2015. Pak3 regulates apical-basal polarity in migrating border cells during Drosophila oogenesis. Development. 142:3692-3703.

Frydman HM, Spradling AC. 2001. The receptor-like tyrosine phosphatase lar is required for epithelial planar polarity and for axis determination within Drosophila ovarian follicles. Development. 128:3209-3220.

Gates J. 2012. Drosophila egg chamber elongation: insights into how tissues and organs are shaped. Fly. 6:213-227.

Genova JL, Fehon RG. 2003. Neuroglian, gliotactin, and the $\mathrm{Na}^{+} / \mathrm{K}^{+}$ ATPase are essential for septate junction function in Drosophila. J Cell Biol. 161:979-989.

Gupta T, Schüpbach T. 2003. Cct1, a phosphatidylcholine biosynthesis enzyme, is required for Drosophila oogenesis and ovarian morphogenesis. Development. 130:6075-6087.

Gutzeit HO, Eberhardt W, Gratwohl E.1991. Laminin and basement membrane-associated microfilaments in wild-type and mutant Drosophila ovarian follicles. J Cell Sci. 100:781-788.

Haigo SL, Bilder D. 2011. Global tissue revolutions in a morphogenetic movement controlling elongation. Science. 331:1071-1074.

Hall S, Bone C, Oshima K, Zhang L, McGraw M, et al. 2014. Macroglobulin complement-related encodes a protein required for septate junction organization and paracellular barrier function in Drosophila. Development. 141:889-898.

Hall S, Ward RE. 2016. Septate junction proteins play essential roles in morphogenesis throughout embryonic development in Drosophila. G3 (Bethesda). 6:2375-2384.

Hayes P, Solon J. 2017. Drosophila dorsal closure: an orchestra of forces to zip shut the embryo. Mech Dev. 144:2-10.

He L, Wang X, Tang HL, Montell DJ. 2010. Tissue elongation requires oscillating contractions of a basal actomyosin network. Nat Cell Biol. 12:1133-1142.

Hildebrandt A, Pflanz R, Behr M, Tarp T, Riedel D, et al. 2015. Bark beetle controls epithelial morphogenesis by septate junction maturation in Drosophila. Dev Biol. 400:237-247.

Horne-Badovinac S. 2020. The Drosophila micropyle as a system to study how epithelia build complex extracellular structures. Philos Trans R Soc Lond B Biol Sci. 375:20190561.

Horne-Badovinac S, Bilder D. 2005. Mass transit: epithelial morphogenesis in the Drosophila egg chamber. Dev Dyn. 232:559-574.

Horne-Badovinac S, Hill J, Gerlach G, Menegas W, Bilder D. 2012. A screen for round egg mutants in Drosophila identifies tricornered, furry, and misshapen as regulators of egg chamber elongation. G3 (Bethesda). 2:371-378.

Ile KE, Tripathy R, Goldfinger V, Renault AD. 2012. Wunen, a Drosophila lipid phosphate phosphatase, is required for septate 
junction-mediated barrier function. Development. 139: 2535-2546.

Isabella AJ, Horne-Badovinac S. 2016. Rab10-mediated secretion synergizes with tissue movement to build a polarized basement membrane architecture for organ morphogenesis. Dev Cell. 38:47-60.

Isasti-Sanchez J, Munz-Zeise F, Lancino M, Luschnig S. 2021. Transient opening of tricellular vertices controls paracellular transport through the follicle epithelium during Drosophila oogenesis. Dev. Cell. 56:1083-1099.

Izumi Y, Furuse M. 2014. Molecular organization and function of invertebrate occluding junctions. Semin Cell Dev Biol. 36:186-193.

Khammari A, Agnès F, Gandille P, Pret AM. 2011. Physiological apoptosis of polar cells during Drosophila oogenesis is mediated by hid-dependent regulation of Diap1. Cell Death Differ. 18:793-805.

Lamb RS, Ward RE, Schweizer L, Fehon RG. 1998. Drosophila coracle, a member of the protein 4.1 superfamily, has essential structural functions in the septate junctions and developmental functions in embryonic and adult epithelial cells. Mol Biol Cell. 9:3505-3519.

Mahowald AP. 1972. Ultrastructural observations on oogenesis in Drosophila. J Morphol. 137:29-48.

Maimon I, Popliker M, Gilboa L. 2014. Without children is required for Stat-mediated Zfh1 transcription and for germline stem cell differentiation. Development. 141:2602-2610.

Montell DJ. 2003. Border-cell migration: the race is on. Nat Rev Mol Cell Biol. 4:13-24.

Moyer KE, Jacobs JR. 2008. Varicose: a MAGUK required for the maturation and function of Drosophila septate junctions. BMC Dev Biol. 8:99.

Müller HA. 2000. Genetic control of epithelial cell polarity: lessons from Drosophila. Dev Dyn. 218:52-67.

Murphy AM, Montell DJ. 1996. Cell type-specific roles for Cdc42, Rac, and RhoL in Drosophila oogenesis. J Cell Biol. 133:617-630.

Nelson KS, Furuse M, Beitel GJ. 2010. The Drosophila claudin kune-kune is required for septate junction organization and tracheal tube size control. Genetics. 185:831-839.

Noirot-Timothée C, Smith DS, Cayer ML, Noirot C. 1978. Septate junctions in insects: comparison between intercellular and intramembranous structures. Tissue Cell. 10:125-136.

Ogienko AA, Yarinich LA, Fedorova EV, Lebedev MO, Andreyeva EN, et al. 2018. New Slbo-Gal4 driver lines for the analysis of border cell migration during Drosophila oogenesis. Chromosoma. 127:475-487.

Oshima K, Fehon RG. 2011. Analysis of protein dynamics within the septate junction reveals a highly stable core protein complex that does not include the basolateral polarity protein discs large. J Cell Sci. 124:2861-2871.

Paul SM, Ternet M, Salvaterra PM, Beitel GJ. 2003. The $\mathrm{Na}^{+} / \mathrm{K}^{+}$ ATPase is required for septate junction function and epithelial tube-size control in the Drosophila tracheal system. Development. 130:4963-4974.

Prasad M, Montell DJ. 2007. Cellular and molecular mechanisms of border cell migration analyzed using time-lapse live-cell imaging. Dev Cell. 12:997-1005.

Qin X, Park B, Liu J, Chen B, Choesmel-Cadamuro V, et al. 2017. Cell-matrix adhesion and cell-cell adhesion differentially control basal myosin oscillation and Drosophila egg chamber elongation. Nat Commun. 8:14708

Schindelin J, Arganda-Carreras I, Frise E, Kaynig V, Longair M, et al. 2012. Fiji: an open-source platform for biological-image analysis. Nat Methods. 9:676-682.

Schneider M, Khalil AA, Poulton J, Castillejo-Lopez C, Egger-Adam D, et al. 2006. Perlecan and dystroglycan act at the basal side of the Drosophila follicular epithelium to maintain epithelial organization. Development. 133:3805-3815.

Schulte J, Tepass U, Auld VJ. 2003. Gliotactin, a novel marker of tricellular junctions, is necessary for septate junction development in Drosophila. J Cell Biol. 161:991-1000.

Snow PM, Bieber AJ, Goodman CS. 1989. Fasciclin III: a novel homophilic adhesion molecule in Drosophila. Cell. 59:313-323.

Spradling AC, de Cuevas M, Drummond-Barbosa D, Keyes L, Lilly M, et al. 1997. The Drosophila germarium: stem cells, germ line cysts, and oocytes. Cold Spring Harb Symp Quant Biol. 62:25-34.

Tepass U, Hartenstein V. 1994. The development of cellular junctions in the Drosophila embryo. Dev Biol. 161:563-596.

Tepass U, Tanentzapf G, Ward R, Fehon R. 2001. Epithelial cell polarity and cell junctions in Drosophila. Annu Rev Genet. 35:747-784.

Tiklová K, Senti KA, Wang S, Gräslund A, Samakovlis C. 2010. Epithelial septate junction assembly relies on melanotransferrin iron binding and endocytosis in Drosophila. Nat Cell Biol. 12: 1071-1077.

VanHook A, Letsou A. 2008. Head involution in Drosophila: genetic and morphogenetic connections to dorsal closure. Dev Dyn. 237: 28-38.

Venema DR, Zeev-Ben-Mordehai T, Auld VJ. 2004. Transient apical polarization of gliotactin and coracle is required for parallel alignment of wing hairs in Drosophila. Dev Biol. 275:301-314.

Viktorinová I, König T, Schlichting K, Dahmann C. 2009. The cadherin Fat2 is required for planar cell polarity in the Drosophila ovary. Development. 136:4123-4132.

Ward RE, Lamb RS, Fehon RG. 1998. A conserved functional domain of Drosophila coracle is required for localization at the septate junction and has membrane-organizing activity. J Cell Biol. 140: 1463-1473.

Wei J, Hortsch M, Goode S. 2004. Neuroglian stabilizes epithelial structure during Drosophila oogenesis. Dev Dyn. 230:800-808.

Wittes J, Schüpbach T. 2019. A gene expression screen in Drosophila melanogaster identifies novel JAK/STAT and EGFR targets during oogenesis. G3 (Bethesda). 9:47-60.

Wu VM, Schulte J, Hirschi A, Tepass U, Beitel GJ. 2004. Sinuous is a Drosophila claudin required for septate junction organization and epithelial tube size control. J Cell Biol. 164:313-323.

Wu VM, Yu MH, Paik R, Banerjee S, Liang Z, et al. 2007. Drosophila varicose, a member of a new subgroup of basolateral MAGUKs, is required for septate junctions and tracheal morphogenesis. Development. 134:999-1009.

Communicating editor: B. H. Reed 